\title{
A randomised clinical trial of nebulised tobramycin or colistin in cystic fibrosis
}

\author{
M.E. Hodson*, C.G. Gallagher", J.R.W. Govan"
}

A randomised clinical trial of nebulised tobramycin or colistin in cystic fibrosis. M.E. Hodson, C.G. Gallagher, J.R.W. Govan. (C)ERS Journals Ltd 2002.

ABSTRACT: Chronic infection with Pseudomonas aeruginosa is associated with progressive deterioration in lung function in cystic fibrosis $(C F)$ patients. The purpose of this trial was to assess the efficacy and safety of tobramycin nebuliser solution (TNS) and nebulised colistin in CF patients chronically infected with $P$. aeruginosa.

One-hundred and fifteen patients, aged $\geqslant 6 \mathrm{yrs}$, were randomised to receive either TNS or colistin, twice daily for 4 weeks. The primary end point was an evaluation of the relative change in lung function from baseline, as measured by forced expiratory volume in one second \% predicted. Secondary end points included changes in sputum $P$. aeruginosa density, tobramycin/colistin minimum inhibitory concentrations and safety assessments.

TNS produced a mean $6.7 \%$ improvement in lung function $(\mathrm{p}=0.006)$, whilst there was no significant improvement in the colistin-treated patients (mean change $0.37 \%$ ). Both nebulised antibiotic regimens produced a significant decrease in the sputum $P$. aeruginosa density, and there was no development of highly resistant strains over the course of the study. The safety profile for both nebulised antibiotics was good.

Tobramycin nebuliser solution significantly improved lung function of patients with cystic fibrosis chronically infected with Pseudomonas aeruginosa, but colistin did not, in this study of 1-month's duration. Both treatments reduced the bacterial load.

Eur Respir J 2002; 20: 658-664.
*Royal Brompton \& Harefield NHS Trust, London, UK. "St Vincent's University Hospital, Dublin, Ireland. "Medical School, University of Edinburgh, Edinburgh, UK.

Correspondence: M.E. Hodson, Dept of Cystic Fibrosis, Royal Brompton \& Harefield NHS Trust, Sydney Street, London SW3 6NP, UK.

Fax: 442073518052

E-mail: s.hockley@ic.ac.uk

Keywords: Colistin

cystic fibrosis

Pseudomonas aeruginosa

tobramycin

Received: May 292001

Accepted after revision: March 202002

This study was sponsored by PathoGenesis Ltd, Hownslow, UK.
Endobronchial infection with Pseudomonas aeruginosa is a characteristic of cystic fibrosis (CF) [1-4] and is closely associated with progressive deterioration in lung function and mortality in adolescents and adults, with patients losing an average of $2 \%$ of their lung function per year [5,6].

The aim of antibiotic therapy in the chronically infected CF patient is to stabilise lung function and, if possible, to restore some of the lost lung function [7-11]. The regular use of nebulised antibiotic therapy in Europe is common for the treatment of lung infections $[12,13]$. This route ensures high antibiotic concentrations at the site of infection while reducing systemic exposure $[11,12,14]$. Nebulised colistin has been shown to reduce the decline in lung function in CF patients compared with placebo, and as a single therapeutic agent it is an established treatment for CF patients with $P$. aeruginosa infection [15].

The clinicians involved in this study have, in general, a policy of treating first isolation of Pseudomonas with ciprofloxacin and inhaled colomycin. All patients are treated for acute exacerbations of infection, and patients with chronic pseudomonal infection are offered maintenance inhaled antibiotics. Colomycin is the drug of first choice, but aminoglycosides are used by some centres to a small degree. There is not a general policy of regular, 3-monthly intravenous antibiotics, although a few patients are receiving this treatment.
Tobramycin nebuliser solution (TNS), a preservativefree formulation for nebulisation, has recently been licensed as a clinically validated drug and delivery system. In the USA, placebo-controlled studies have demonstrated significant improvements in lung function within 14 days for TNS-treated patients $[16,17]$ and that lung function remained above baseline during longterm intermittent treatment cycles (4 weeks on/4 weeks off) for 18-24 months [18, 19].

In the present study, CF patients infected with $P$. aeruginosa received a 4-week, twice-daily aerosol administration of either TNS or nebulised colistin. The primary objective of the study was to evaluate the change in lung function, as measured by forced expiratory volume in one second (FEV1) \% predicted, with secondary objectives including an evaluation of the change in sputum $P$. aeruginosa density and changes in minimum inhibitory concentrations (MICs) of tobramycin and MICs of colistin against $P$. aeruginosa in the two treatment groups.

\section{Methods}

\section{Patients}

Eligible patients were enrolled from $16 \mathrm{CF}$ centres in the UK and Ireland. Inclusion criteria were as follows: age of $\geqslant 6 \mathrm{yrs}$; documented diagnosis of 
cystic fibrosis (sweat chloride $\geqslant 60 \mathrm{mEq} \cdot \mathrm{L}^{-1}$ by quantitative pilocarpine iontophoresis test, sweat sodium $\geqslant 70 \mathrm{mEq} \cdot \mathrm{L}^{-1}$, homozygosity for $\Delta \mathrm{F}_{508}$ genetic mutation, or heterozygosity for two well-characterised mutations); FEV $1 \geqslant 25 \%$ pred (using equations from KNUDSON et al. [20]); $P$. aeruginosa present in a sputum or throat culture within the previous 12 months and in the sputum at the screening visit; ability to perform lung function tests and to expectorate sputum. Patients were allowed to continue their routine nonantipseudomonal medications and physiotherapy regimens. Therapy with recombinant human deoxyribonuclease (rhDNase), provided it had been started at least 4 weeks prior to screening, could also be continued.

Patients were excluded if they had received antipseudomonal antibiotics by any route within the previous 14 days or any investigational drug within 4 weeks prior to initial study drug administration. Also excluded were patients with the following: known local or systemic hypersensitivity to aminoglycosides or polymyxins, significant haemoptysis or new changes on chest radiograph, pregnancy, impaired renal function, or a sputum or throat culture yielding Burkholderia cepacia within the previous 2 yrs.

The study protocol was approved by the North Thames Multicentre Research Ethics Committee and local ethics committees at each of the participating centres. Written informed consent was obtained from all patients or, in the case of patients aged $<18 \mathrm{yrs}$, from their legal guardian.

\section{Study design and treatment regimen}

Following completion of a 2-week screening period, during which eligibility was determined and no antipseudomonal drugs administered, suitable patients were randomised into the two parallel treatment groups. Randomisation was stratified by age (6-12 yrs, $13-17$ yrs, $\geqslant 18$ yrs) and centre.

Patients received either TNS $300 \mathrm{mg} \cdot 5 \mathrm{~mL}^{-1}$ (tobramycin solution for inhalation (TOBI) $\mathbb{R}$, PathoGenesis Ltd, Hownslow, UK) or colistin sulphomethate

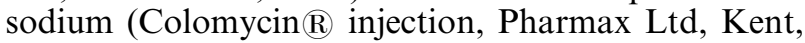
UK) $80 \mathrm{mg}$ dissolved in $3 \mathrm{~mL}$ of preservative-free normal saline, by inhalation twice daily for 4 weeks. TNS was administered using the PARI LC PLUS ${ }^{\text {TM }}$ nebuliser (Pari Medical Ltd, West Byfleet, UK) and CR50 compressor (Medic-Aid, Bognor Regis, UK). Colistin was administered using the Ventstream ${ }^{\text {TM }}$ nebuliser (Medic-Aid) and the CR50 compressor (Medic-Aid).

Clinical evaluations and spirometry were performed, and sputum samples obtained for microbiology at screening (week -2), baseline (week 0) and week 4. Clinical evaluation by the investigators and patients was appraised by assessing global improvement using the Global Rating of Change [21]. Lung function was measured by spirometry [22].

The primary efficacy end point of the study was the mean change from baseline to week 4 in FEV1\% pred. The mean relative $\%$ change in FEV1 \% pred was calculated using the following formula:

$$
\begin{aligned}
& \frac{\text { FEV }_{1} \% \operatorname{pred}_{\text {week } 4}-\mathrm{FEV}_{1} \% \operatorname{pred}_{\text {week } 0}}{\text { FEV } 1_{1} \% \text { pred }_{\text {week } 0}} \times 100 \\
& =\% \text { change in FEV } 1 \% \text { pred }
\end{aligned}
$$

Sputum samples were shipped on wet ice to the central laboratory (Dept of Medical Microbiology University of Edinburgh Medical School, Edinburgh, UK) and processed at the laboratory within $48 \mathrm{~h}$ of collection. Samples were cultured at baseline and week 4 to assess the following microbiological parameters: sputum $P$. aeruginosa density ( $\log _{10}$ colony forming units (cfu)), antimicrobial susceptibility, and incidence of recovery of other microbial pathogens [23].

Patients were asked to report any adverse events occurring during the study period. Any new adverse events occurring during the treatment period were followed up until resolution or until week 8. Airway reactivity (bronchospasm) was assessed by measuring FEV1 before and $30 \mathrm{~min}$ after the first and last dose of study drug administration. Serum creatinine, urea by blood urea nitrogen (BUN) and a urine protein dipstick test were performed at screening and end of treatment, but repeated at the other visits if previous findings were abnormal.

A retrospective examination was made of all the case notes to discover how many patients had received intravenous or inhaled TNS or colomycin in the 6 months prior to the study.

\section{Statistical analysis}

A target sample size of 60 per group was chosen to yield $80 \%$ coverage probability of the $95 \%$ confidence intervals, with length $6.5 \%$ on either side of the observed mean relative change in FEV1 \% pred for the tobramycin group.

Efficacy analyses were completed for intent-to-treat (ITT) patients, defined as patients who had received at least one dose of study medication and had FEV1 measurements at baseline and at week 4 .

The analysis of change in sputum $P$. aeruginosa density $\left(\mathrm{cfu} \cdot \mathrm{mL}^{-1}\right)$ was also performed for a microbiologically evaluable population consisting of patients who had received at least one dose of study medication and had microbiologically evaluable sputum samples at baseline and at week 4 .

Demographics and baseline characteristics were compared using the paired t-test or Chi-squared test. The analyses of relative change in FEV1\% pred were performed as Wilcoxon signed-rank tests. The initial statistical analysis plan only included within-group comparisons for the efficacy parameters. However, subsequent between-group comparisons for the lung function end points were conducted using the Wilcoxon rank-sum test.

Changes in sputum $P$. aeruginosa density were analysed using the paired t-test. The treatment groups' MICs were compared using the CochranMantel-Haenszel test, controlled by centre. Airway 


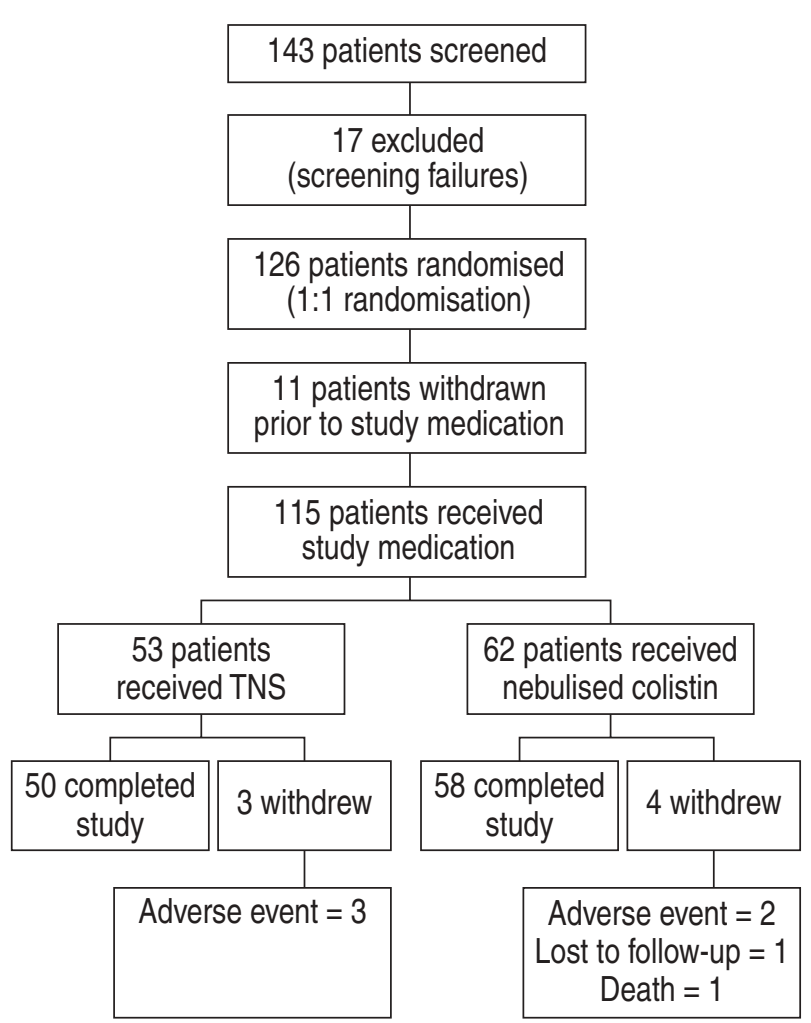

Fig. 1. - Trial profile. TNS: tobramycin nebuliser solution.

reactivity was analysed within each group using a paired t-test.

\section{Results}

The study was completed through to follow-up at week 8 in 50 of 53 patients in the TNS-treatment group $(94.3 \%)$ and 58 of 62 patients in the colistintreatment group $(93.5 \%)$ (fig. 1). Treatment compliance, defined as use of $\geqslant 75 \%$ of the dispensed ampoules, was $98.1 \%$ in the TNS-treatment group and $87.1 \%$ in the colistin-treatment group.

The patient groups were similar at baseline with respect to age, sex, rhDnase usage, lung function and sputum $P$. aeruginosa density (table 1 ).

\section{Lung function}

Within the TNS-treatment group, there was a highly significant $6.70 \%$ increase $(\mathrm{p}=0.006)$ in FEV1 $\%$ pred from baseline to week 4 in the ITT patient population; whereas, within the colistin-treatment group, the $0.37 \%$ change in FEV1 \% pred was not significantly different from baseline (table 2). Furthermore, the increase in FEV1 \% pred was significantly larger in the TNS-treatment group when compared to the colistin-treatment group ( $\mathrm{p}=0.008)$. When the $\%$ change in FEV1 \% pred was analysed according to patient age, TNS treatment was found to be especially beneficial for younger patients (table 2).

At week 4, investigators assessed the change in the patients' general medical condition through the completion of a Global Rating of Change questionnaire. More TNS-treated patients (21 of 53, 39.6\%) than colistin-treated patients (10 of $62,16.1 \%$ ) were assessed as having an improved medical condition ( $p=0.006$ Fisher's exact test). Similarly, more patients in the TNS-treatment group (13 of 53, 24.5\%) rated themselves as improved at the end of the treatment period, as compared to those treated with colistin ( 8 of $62,12.9 \%)$.

\section{Microbiology}

In the ITT population at week 4 , a mean decrease of $0.86 \log _{10} \mathrm{cfu} \cdot \mathrm{mL}^{-1}$ was observed in TNStreated patients $(\mathrm{p}<0.001)$, and a mean decrease of $0.60 \log _{10} \mathrm{cfu} \cdot \mathrm{mL}^{-1}$ was observed in colistin-treated patients $(\mathrm{p}=0.007)$ (table 3$)$.

To take account of the heterogeneity of MIC values commonly observed with $P$. aeruginosa infections in $\mathrm{CF}$ airways, the authors determined the percentage of patients whose highest MIC of tobramycin against $P$. aeruginosa (based on analysis of different colonial morphotypes) was $\geqslant 4 \mathrm{mg} \cdot \mathrm{L}^{-1}$ at baseline and/or week 4. After 4 weeks of TNS treatment, there was a

Table 1.-Baseline characteristics of patients

\begin{tabular}{|c|c|c|c|}
\hline Characteristic & TNS & Colistin & p-value \\
\hline Patients $n$ & 53 & 62 & \\
\hline \multicolumn{4}{|l|}{ Sex } \\
\hline Male & $20(37.7)$ & $32(51.6)$ & \multirow[t]{2}{*}{$0.136^{\#}$} \\
\hline Female & $33(62.3)$ & $30(48.4)$ & \\
\hline \multicolumn{4}{|l|}{ Age yrs } \\
\hline Mean \pm SD & $21.3 \pm 9.6$ & $20.1 \pm 9.4$ & \multirow[t]{2}{*}{$0.505^{\bullet}$} \\
\hline Median (range) & $20.0(7-42)$ & $18.0(8-50)$ & \\
\hline DNase use & $36(67.9)$ & $34(45.2)$ & ND \\
\hline Nebulised antibiotic in previous 6 months $\#$ & $48(90.6)$ & $50(80.6)$ & ND \\
\hline FEV $1 \%$ pred & $55.4 \pm 22.9$ & $59.4 \pm 22.6$ & $0.349^{\bullet}$ \\
\hline FVC $\%$ pred & $72.5 \pm 21.7$ & $76.40 \pm 19.2$ & $0.327^{\star}$ \\
\hline $\log _{10} \mathrm{cfu} \cdot \mathrm{mL}^{-1+}$ & $6.30 \pm 1.52$ & $6.77 \pm 1.34$ & $0.077^{\bullet}$ \\
\hline
\end{tabular}

Data are presented as mean \pm SD and n (\%) unless otherwise stated. DNase: deoxyribonuclease; TNS: tobramycin nebuliser solution; FEV1: forced expiratory volume in one second; FVC: forced vital capacity; ND: not done; cfu: colony forming unit. \#: Chi-squared test; ${ }^{\text {I: }}$ : paired t-test; ${ }^{+}$: sputum Pseudomonas aeruginosa density. 
Table 2. - Change in lung function following 4 weeks of nebulised antibiotic therapy

\begin{tabular}{|c|c|c|c|}
\hline \multirow[t]{2}{*}{ Assessment } & \multicolumn{2}{|c|}{ FEV1 $\%$ pred $\%$ change from baseline } & \multirow[t]{2}{*}{ p-value } \\
\hline & TNS & Colistin & \\
\hline \multicolumn{4}{|l|}{ ITT population } \\
\hline Patients at week $4 \mathrm{n}$ & 50 & 59 & \\
\hline Mean \pm SD & $6.70 \pm 15.12$ & $0.37 \pm 18.78$ & 0.008 \\
\hline p-value (within) $)^{\#}$ & 0.006 & 0.473 & \\
\hline \multicolumn{4}{|l|}{ Age group 6-12 } \\
\hline Patients at week $4 \mathrm{n}$ & 11 & 11 & \\
\hline Mean \pm SD & $11.51 \pm 24.33$ & $-8.11 \pm 18.38$ & \\
\hline p-value (within) & 0.148 & 0.17 & \\
\hline \multicolumn{4}{|l|}{ Age group 13-17 } \\
\hline Patients at week $4 \mathrm{n}$ & 11 & 14 & \\
\hline Mean \pm SD & $14.43 \pm 7.32$ & $6.01 \pm 25.78$ & \\
\hline p-value (within) & $<0.001$ & 0.399 & \\
\hline \multicolumn{4}{|l|}{ Age group $>18$} \\
\hline Patients at week $4 \mathrm{n}$ & 28 & 34 & \\
\hline Mean \pm SD & $1.77 \pm 10.80$ & $0.79 \pm 14.75$ & \\
\hline p-value (within) ${ }^{\#}$ & 0.393 & 0.757 & \\
\hline
\end{tabular}

TNS: tobramycin nebuliser solution; ITT: intent-to-treat; FEV1: forced expiratory volume in one second. ${ }^{*}$ : within-group paired t-testing; ${ }^{\uparrow}$ : between-group testing using Wilcoxon rank-sum test.

nonsignificant increase in the percentage of patients with at least one $P$. aeruginosa isolate, with a tobramycin MIC value $\geqslant 4 \mathrm{mg} \cdot \mathrm{L}^{-1}$ from $38 \%$ to $49 \%$; whereas, in the colistin-treatment group, it remained unchanged at 55\%. After 4 weeks of TNS treatment, there was a nonsignificant decrease in the percentage of patients whose $P$. aeruginosa had colistin MIC values $\geqslant 4 \mathrm{mg} \cdot \mathrm{L}^{-1}$ from $27 \%$ to $16 \%$; whereas, in the colistin treatment group, it remained unchanged at $34 \%$. There was no evidence of the development of highly tobramycin-resistant $P$. aeruginosa (MIC $\geqslant 128 \mathrm{mg} \cdot \mathrm{L}^{-1}$ ) in either treatment group over the duration of the study.

No patients in either treatment group became colonised with either B. cepacia or Stenotrophomonas maltophilia during the 4 weeks of the study. The organisms that were isolated more frequently in the TNS group than in the colistin group following treatment were Candida albicans (11.3\% versus $4.8 \%)$

Table 3.-Change in sputum Pseudomonas aeruginosa density following 4 weeks of nebulised antibiotic therapy

\begin{tabular}{lcc}
\hline $\log _{10} \mathrm{cfu} \cdot \mathrm{mL}^{-1}$ & \multicolumn{2}{c}{ Change from baseline } \\
\cline { 2 - 3 } & TNS & Colistin \\
\hline ITT population & & \\
Patients assessed & 50 & 50 \\
at week 4 $\mathrm{n}$ & $-0.86 \pm 1.43$ & $-0.60 \pm 1.61$ \\
Mean \pm SD & $<0.001$ & 0.007 \\
p-value (within) & & \\
Microbiologically-evaluable & & 37 \\
patients & 42 & $-0.47 \pm 1.53$ \\
Assessed at week 4 n & $-0.79 \pm 1.35$ & 0.049 \\
Mean \pm SD & $<0.001$ & \\
p-value (within) & &
\end{tabular}

TNS: tobramycin nebuliser solution; cfu: colony forming unit; ITT: intent-to-treat. ${ }^{\#}$ : between-group testing using the Wilcoxon rank-sum test. and Aspergillus fumigatis (5.7\% versus 3.2\%). Colistin treatment more frequently resulted in colonisation with Haemophilus influenzae $(8.1 \%$ versus $1.9 \%)$ and Staphylococcus aureus $(6.5 \%$ versus $0 \%)$.

\section{Adverse events}

The incidence of adverse events was comparable in the TNS and colistin groups (table 4). Thirty-four of 53 patients $(64 \%)$ in the TNS-treatment group and 31 of 62 patients $(50 \%)$ in the colistin-treatment group reported at least one treatment-emergent adverse event. For both treatment groups, the highest incidence of adverse events related to the respiratory system and to the "body as a whole". Twenty-six of $53(49 \%)$ TNS patients and 22 of $62(36 \%)$ colistin patients reported at least one respiratory system adverse event; pharyngitis was the most common treatment-emergent event in the TNS-treatment

Table 4. - Incidence of adverse events (AE)

\begin{tabular}{lcc}
\hline & TOBI & Colistin \\
\hline Patients n & 53 & 62 \\
Patients with $\geqslant 1$ & $34(64.2)$ & $31(50.0)$ \\
$\quad$ treatment-emergent AE & & \\
Respiratory system AE $^{\#}$ & & \\
Total $^{\#}$ & $26(49.1)$ & $22(35.5)$ \\
Cough-increased $^{\#}$ & $5(9.4)$ & $11(17.7)$ \\
Sputum-increased $^{\#}$ & $6(11.3)$ & $8(12.9)$ \\
Dyspnoea $^{\#}$ & $5(9.4)$ & $7(11.3)$ \\
Pharyngitis $^{\#}$ & $7(13.2)$ & $3(4.8)$ \\
Patients with $^{*}$ & $8(15.1)$ & $7(11.3)$ \\
serious AE $^{\#}$ & & \\
\hline
\end{tabular}

Data are presented as $\mathrm{n}(\%)$. TOBI: tobramycin solution for inhalation. " : no significant difference was detected between groups. 
Table 5. - Acute change in forced expiratory volume in one second (FEV 1 ) from pre- to 30 min post-administration of the study drug at baseline and week 4

\begin{tabular}{lcc}
\hline$\%$ Change in FEV1 & TNS & Colistin \\
\hline Week 0 & 51 & \\
Patients n & $-4.49 \pm 8.11$ & $-3.87 \pm 9.36$ \\
Mean \pm SD & $<0.001$ & $<0.001$ \\
p-value & & \\
Week 4 & 48 & 58 \\
Patients $\mathrm{n}$ & $-2.57 \pm 5.39$ & $-2.59 \pm 9.02$ \\
Mean \pm SD & 0.002 & 0.030 \\
p-value & & \\
\hline
\end{tabular}

TNS: tobramycin nebuliser solution. ${ }^{*}$ : within-group testing using the paired t-test.

group and increased cough in the colistin-treatment group.

There were no clinically significant changes in renal function over the 4-week treatment period in either group.

\section{Airway reactivity}

Airway reactivity is an expected response to the administration of nebulised antibiotics in some $\mathrm{CF}$ patients. In this study, $85 \%$ of patients were receiving $\beta_{2}$-adrenoreceptor agonists. The acute change in FEV1 (from pre- to $30 \mathrm{~min}$ post-administration of study drug) at baseline and week 4 showed significant falls in both treatment groups, with a degree of tolerance developing over the treatment period (table 5). Airway reactivity, as defined by a $\geqslant 10 \%$ loss in FEV1 30 min after nebulisation of the study drug, was recorded in 6 of $53(11.3 \%)$ patients in the TNS-treatment group and 11 of $62(17.7 \%)$ patients in the colistin-treatment group.

\section{Previous exposure to tobramycin or colistin}

Twenty-five of $53(47 \%)$ TNS-treated patients had received nebulised or intravenous tobramycin in the 6 months before the study. Comparable figures for colistin were 51 of $62(82 \%)(\mathrm{p}=0.0001$, Fisher's exact test) (table 6).

\section{Discussion}

The results of this study demonstrated that TNS produced a significant improvement in lung function as well as a significant decrease in sputum $P$. aeruginosa density in chronically infected CF patients. Nebulised colistin produced a significant decrease in sputum $P$. aeruginosa density, but did not significantly improve lung function.

A double-blind trial design could not be followed for this study for a number of reasons. Colistin requires reconstitution with saline before administration and has a tendency to foam, whereas TNS is supplied as a liquid in ampoules and has a very distinctive taste. Blinding was further complicated by the use of different breath enhanced nebulisers; TNS was administered via the PARI LC PLUS ${ }^{\mathrm{TM}}$ (the approved nebuliser; Pari Medical Ltd) and colistin was administered via the Ventstream ${ }^{\mathrm{TM}}$ nebuliser (Medic-Aid), as a pre-trial survey identified this as the most commonly chosen nebuliser for this purpose in current clinical practice. A majority of patients had received nebulised colistin in the 6 months prior to this study, and hence would likely be aware of which treatment they were receiving. The absence of a double-blind trial means that the self-assessments by the patients and the clinical global assessments by the investigators could have been biased because of awareness of receiving a "new treatment". However, the key primary and secondary end points of this study were objective.

The study utilised the approved dosage regimen and nebuliser for TNS. The choice of colistin treatment regimen (1 megaunit b.i.d.) was consistent with current clinical practice [13] and the recommended British National Formulary dosage. It was also the dose received by $80 \%$ of patients prescribed colistin therapy in the 6 months prior to study start. However, a few centres in the UK and Denmark do routinely use 2 megaunits b.i.d. It could be suggested that the lack of improvement in this study in the colistin treatment arm was due to previous exposure to colistin. However, although only a few of the patients had previously received inhaled aminoglycosides, most of them would have received intravenous gentamicin or tobramycin.

The baseline demographics of both groups were similar (table 1). Although there was no statistically significant difference, more patients in the TNS group received dornase alfa (Pulmozyme $\mathbb{R}$, Genentech, Inc.,

Table 6. - Previous exposure to tobramycin and colistin

\begin{tabular}{|c|c|c|c|c|c|c|c|}
\hline \multirow[t]{2}{*}{ Patients randomised to } & \multirow[t]{2}{*}{ Time before treatment } & \multicolumn{3}{|c|}{ Tobramycin } & \multicolumn{3}{|c|}{ Colistin } \\
\hline & & Inhaled & i.v. & Either & Inhaled & i.v. & Either \\
\hline \multicolumn{8}{|l|}{ TOBI $^{\#}$} \\
\hline & 1 month & 2 & 8 & 9 & 40 & 1 & 41 \\
\hline \multirow{3}{*}{ Colistin } & 6 months & 3 & 22 & 25 & 43 & 4 & 45 \\
\hline & 1 month & 3 & 6 & 9 & 48 & 2 & 50 \\
\hline & 6 months $^{+}$ & 3 & 26 & 29 & 50 & 3 & 51 \\
\hline
\end{tabular}

TOBI: tobramycin solution for inhalation. ${ }^{\#}: \mathrm{n}=53 ;{ }^{\uparrow}: \mathrm{n}=62 ;{ }^{+}$: data on one patient missing due to lost notes. 
San Francisco, CA, USA) than in the colistin-treated group. It is possible that dornase alfa enhances the penetration of the antibiotic to the bronchial epithelium.

The lack of improvement in FEV1\% pred observed for the colistin group is consistent with a previous study of nebulised colistin. A placebo-controlled study of 40 Danish patients given either nebulised colistin (1 megaunit) or placebo twice daily for 30 days showed that while the FEV1\% pred declined in both treatment groups, the decline in the colistin treatment arm was less than that observed for the placebo arm [15].

The sample size of this trial was based on demonstrating within-group significance for the treatment groups. However, the between-group differences proved to be statistically significant, favouring the TNS-treatment group.

The improvement in FEV1\% pred seen in TNStreated patients is also consistent with previous studies of nebulised tobramycin. Two double-blind, placebocontrolled clinical trials of TNS (300 mg tobramycin b.i.d.) delivered via the PARI LC PLUS ${ }^{\text {TM }}$ nebuliser (Pari Medical Ltd) to $520 \mathrm{CF}$ patients in the USA showed that TNS resulted in a $11.9 \%$ improvement in FEV1 \% pred after 28 days of treatment [17]. In addition, an earlier crossover study of $71 \mathrm{CF}$ patients in the USA given $600 \mathrm{mg}$ tobramycin three times daily via ultrasonic nebuliser produced an absolute increase in $\mathrm{FEV}_{1} \%$ pred of 9.7 percentage points higher than for placebo [24]. In the 1993 study, RAMSEY et al. [24] required patients to be within $10 \%$ of their best forced vital capacity measurement in the previous 6 months at enrolment or 2 weeks after completing intravenous therapy for a pulmonary exacerbation; none of the other studies had this requirement.

Young patients with better preserved function benefited most. This has been seen in other studies of inhaled antibiotics and dornase alfa and may be due to better penetration of inhaled treatment in patients with more preserved lung function.

Both TNS and colistin significantly decreased sputum $P$. aeruginosa density after 4 weeks of treatment (table 3); however, the less than one $\log _{10}$ decrease observed for both treatment groups is less than half the reduction than that observed in the earlier studies of nebulised tobramycin described above [17]. Notably, density of $P$. aeruginosa in sputum at baseline was also higher in these studies than in the current study. In the present study, the small but significant change in cfu may not fully account for the improvement in lung function. As previously suggested [17], in addition to bactericidal activity, inhaled antibiotics may have antiinflammatory effects and reduce production of bacterial virulence factors.

The differences in the magnitude of the improvement in FEV1 \% pred, the reduction of $P$. aeruginosa and baseline $P$. aeruginosa sputum density in these various studies may be due to national/regional differences in the antibiotic therapies patients received prior to enrolling in the studies. In particular, most $\mathrm{CF}$ patients in the UK and Ireland receive nebulised antibiotic as part of their standard care; in the present study, 98 patients $(85 \%)$ had received aerosol antibiotics in the previous 6 months. In the USA, nebulised antibiotics were used much less frequently prior to TOBI registration [25]. In the present study, the greatest benefit from TNS treatment was seen in children and young adults, a finding that has previously been documented [17].

Baseline resistance was present for both tobramycin and colistin at the parenteral break-point levels $\left(\geqslant 4 \mu \mathrm{g} \cdot \mathrm{mL}^{-1}\right)$. A small increase in tobramycin MIC values was noted for TNS-treated patients, while there was no change in colistin MIC values for colistintreated patients. However, there was no relationship between baseline MICs and improvement in lung function (FEV1 \% pred) for either tobramycin or colistin (data not shown). Parenteral break-points are of limited clinical relevance for nebulised therapy in CF patients, since nebulisation ensures high antibiotic concentrations at the site of infection [11, 14, 17].

Both nebulised antibiotic regimens were well tolerated in these patients and $94 \%$ of randomised patients completed the study treatment. Since 48 of the 62 $(77 \%)$ colistin-treated patients had recently received prior nebulised colistin therapy, there may have been some under-reporting of events in this group due to familiarity with the product and because patients intolerant of colistin's adverse effects would have been excluded from entry into the study. The results of serum chemistry, urine protein and airway reactivity did not show any clinically significant adverse effects of either study drug.

In conclusion, in this short-term study, tobramycin nebuliser solution significantly improved the lung function of cystic fibrosis patients chronically infected with Pseudomonas aeruginosa, but colistin did not. Both treatments significantly reduced the bacterial load, although both groups had had prior exposure to both intravenous and nebulised antibiotics in different amounts. The improvement in lung function with tobramycin nebuliser solution was more apparent in children and young adults. Both nebulised antibiotics had equivalent and acceptable safety profiles. The statistically significant between-group differences will lend support to the choice of tobramycin nebuliser solution when optimising antibiotic regimens in the management of chronic pulmonary infection due to Pseudomonas aeruginosa in cystic fibrosis patients who are not showing improvement with conventional therapy including inhaled colistin.

\footnotetext{
Acknowledgements. The authors wish to acknowledge the following co-authors: D. Adeboyeku, Royal Brompton Hospital, London, UK; S. Barry, St Vincent's Hopsital, Dublin, Ireland; D. Bilton, Papworth Hospital, Cambridge, UK; M. Carroll, Southampton General Hospital, Southampton, UK; S. Conway, C. Etherington, Seacroft Hospital and St James' University Hospital, Leeds, UK; R. Dinwiddie, S. Cunningham, Great Ormond Street Hospital for Children, London, UK; S. Elborn, Belfast City Hospital, Belfast, UK; P. Greally, National Children's Hospital, Dublin, Ireland; E. Redmond, M. Mitchell, Royal Belfast Hospital for Sick Children, Belfast, UK; D. Stableforth, Birmingham Heartlands Hospital, Birmingham,
} 
UK; M. Super, D. Corbett, Royal Manchester Children's Hospital, Manchester, UK; C.J. Taylor, O. Pirzada, The Sheffield Children's Hospital, Sheffield, UK; A. Thomson, John Radcliffe Hospital, Oxford, UK; K. Webb, A Verma, Wythenshawe Hospital, Manchester, UK; P. Weller, Birmingham Children's Hospital, Birmingham, UK.

\section{References}

1. Hoiby N. Cystic fibrosis and endobronchial Pseudomonas infection. Curr Opin Pediatr 1993; 5: 247-254.

2. Denton M, Wilcox MH. Antimicrobial treatment of pulmonary colonization and infection by Pseudomonas aeruginosa in cystic fibrosis patients. $J$ Antimicrob Chemother 1997; 40: 468-474.

3. Govan JRW, Deretic V. Microbial pathogenesis in cystic fibrosis: mucoid Pseudomonas aeruginosa and Burkholderia cepacia. Microbiol Rev 1996; 60: 539574.

4. Fitzsimmons SC. The changing epidemiology of cystic fibrosis. J Pediatr 1993; 122: 1-9.

5. Clinical Guidelines for Cystic Fibrosis Care Working Party Recommendations. Cystic Fibrosis Trust UK. British Paediatric Association and British Thoracic Society, July 1996.

6. Henry RL, Mellis CM, Petrovic L. Mucoid Pseudomonas aeruginosa is a marker of poor survival in cystic fibrosis. Pediatr Pulmonol 1992; 12: 158-161.

7. Kerem E, Reisman J, Corey M, Canny GL, Levison $\mathrm{H}$. Prediction of mortality in patients with cystic fibrosis. N Engl J Med 1992; 326: 1187-1191.

8. Connett GJ. Respiratory management. In: Hill CM, ed. Practical Guidelines for Cystic Fibrosis Care. Philadelphia, Churchill Livingstone, 1998; pp. 21-35.

9. Frederiksen B, Koch C, Hoiby N. Antibiotic treatment of initial colonization with Pseudomonas aeruginosa postpones chronic infection and prevents deterioration of pulmonary function in cystic fibrosis. Pediatr Pulmonol 1997; 23: 330-335.

10. Mendelman PM, Smith AL, Levy J, Weber A, Ramsey B, Davis RL. Aminoglycoside penetration, inactivation, and efficacy in cystic fibrosis sputum. $\mathrm{Am}$ Rev Respir Dis 1985; 132: 761-765.

11. Saiman L. Use of aerosolized antibiotics in patients with cystic fibrosis. Pediatr Infect Dis J 1998; 17: 158159.

12. Touw DJ, Brimcombe RW, Hodson ME, Heijerman HGM, Bakker W. Inhalation of antibiotics in cystic fibrosis. Eur Respir J 1995; 8: 1594-1604.
13. Wyatt H, Ruiz G, Wade J, et al. Guidelines for antimicrobial prescribing in cystic fibrosis. London, Regional Cystic Fibrosis Service, King's Healthcare NHS Trust, 1996.

14. Webb AK, Dodd ME. Nebulised antibiotics for adults with cystic fibrosis. Thorax 1997; 52: Suppl. 2, S69S71.

15. Jensen T, Pedersen SS, Garne S, Heilmann C, Hoiby $\mathrm{N}$, Koch $\mathrm{C}$. Inhalation therapy in cystic fibrosis patients with chronic Pseudomonas aeruginosa lung infection. J Antimicrob Chemother 1987; 19: 831-838.

16. PathoGenesis Corporation. A phase III placebocontrolled clinical trial to study the safety and efficacy of tobramycin solution for inhalation in patients with cystic fibrosis: PC-TNDS-002. Seattle, WA, PathoGenesis Corporation, 1997.

17. Ramsey BW, Pepe MS, Quan JM, Otto KL, Montgomery AB, Williams-Warren J. Intermittent administration of inhaled tobramycin in patients with cystic fibrosis. N Engl J Med 1999; 340: 23-30.

18. Ramsey BW, Quan JM, Otto KL, et al. Long-term efficacy and safety of inhaled tobramycin (TOBI) in patients with cystic fibrosis. Proceedings of the 12th Annual North American Cystic Fibrosis Conference, Montreal, Quebec, Canada, October 15-18, 1998. Pediatr Pulmonol 1998; 26: Suppl. 17, A243.

19. Ramsey BW, Schaeffler B, Montgomery AB, et al. Survival and lung function during two years of treatment with intermittent tobramycin solution for inhalation in $\mathrm{CF}$ patients. Abstract from the 23rd European Cystic Fibrosis Conference, The Hague, The Netherlands, June 9-12, 1999.

20. Knudson RJ, Lebowitz AL, Holberg CJ, Burrows B. Changes in the normal maximal expiratory flowvolume curve with growing and aging. Am Rev Respir Dis 1983; 127: 725-734.

21. Jaeschke R, Singer J, Guyatt GH. Measurement of health status: ascertaining minimal clinically important difference. Control Clin Trials 1989; 10: 407-415.

22. American Thoracic Society. Standardization of spirometry, 1994 update. Am Rev Respir Crit Care Med 1995; 152: 1107-1136.

23. Burns JL, Emerson J, Stapp JR, Yim DL, Krzewinski J, Louden L. Microbiology of sputum from patients at cystic fibrosis centers in the United States. Clin Infect Dis 1998; 27: 158-163.

24. Ramsey BW, Dorking HL, Eisenberg JD, Gibson RL, Harwood IR, Kravitz RM. Efficacy of aerosolised tobramycin in patients with cystic fibrosis. $N$ Engl J Med 1993; 328: 1740-1746.

25. Cystic Fibrosis Foundation Annual Report. Bethesda, MD, Cystic Fibrosis Foundation, 1998. 